\title{
Cisplatin or carboplatin for advanced non-small cell lung cancer: does it really matter?
}

\author{
Meghana Kesireddy ${ }^{1 \wedge}$, Apar Kishor Ganti ${ }^{\wedge}$ \\ ${ }^{1}$ University of Nebraska Medical Center, Omaha, NE, USA; ${ }^{2}$ VA Nebraska Western Iowa Health Care System and University of Nebraska Medical \\ Center, Omaha, NE, USA \\ Correspondence to: Apar Kishor Ganti, MD, MS. Staff Physician, VA Nebraska Western Iowa Health Care System, Professor of Medicine, Division \\ of Oncology-Hematology, Professor (Courtesy) of Biochemistry and Molecular Biology, University of Nebraska Medical Center, 986840 Nebraska \\ Medical Center, Omaha, NE 68198-6840, USA. Email: aganti@unmc.edu. \\ Comment on: Udagawa H, Sugiyama E, Harada T, et al. Bevacizumab plus platinum-based chemotherapy in advanced non-squamous non-small-cell \\ lung cancer: a randomized, open-label phase 2 study (CLEAR). Transl Lung Cancer Res 2021;10:3059-70.
}

Submitted Sep 03, 2021. Accepted for publication Sep 16, 2021.

doi: $10.21037 /$ tlcr-21-718

View this article at: https://dx.doi.org/10.21037/tlcr-21-718

The treatment of non-small cell lung cancer (NSCLC) has undergone a dramatic change in the past few years. In the first-line setting, targeted therapy has replaced cytotoxic chemotherapy in patients with targetable/driver mutations; single-agent immunotherapy has replaced cytotoxic chemotherapy in patients with programmed death ligand 1 (PD-L1) tumor proportion score (TPS) $\geq 50 \%$ in patients with NSCLC including non-squamous NSCLC. Platinum-based doublet chemotherapy combined with immunotherapy and bevacizumab in certain instances, is the mainstay of treatment in the remaining patients.

Keynote 189 was a landmark phase 3 trial that led to the current standard of platinum-based doublet chemotherapy plus immunotherapy (anti-PD-1/anti-PD-L1) in patients with advanced non-squamous NSCLC. This trial showed that the addition of pembrolizumab to platinum (carboplatin was the platinum drug used in $72.2 \%$ of patients) and pemetrexed induction followed pembrolizumab and pemetrexed maintenance increased 12-month overall survival (OS) and median OS compared to placebo. This statistically significant improvement in progression free survival (PFS) and OS with the addition of pembrolizumab was seen across all PD-L1 categories (TPS $<1 \%, 1-49 \%$, $\geq 50 \%)(1)$.

Bevacizumab plus platinum-based doublet chemotherapy is an option for patients with advanced non-squamous
NSCLC. The phase 3 ECOG4599 trial showed that the addition of bevacizumab to carboplatin and paclitaxel chemotherapy (CarPacBev) followed by bevacizumab maintenance improved PFS by 1.7 months and OS by 2 months compared to carboplatin and paclitaxel (CarPac) (2). In the 2000s pemetrexed became the agent of choice for patients with adenocarcinoma as it was shown to be superior to existing agents in multiple studies (3). The PointBreak trial compared pemetrexed based chemotherapy in combination with bevacizumab, i.e., pemetrexed + carboplatin + bevacizumab (CarPemBev) followed by maintenance pemetrexed + bevacizumab to the ECOG4599 regimen. The PointBreak trial did not meet its primary endpoint of improved OS with CarPemBev (median OS 12.6 months in CarPemBev vs. 13.4 months in CarPacBev, HR 1, $\mathrm{P}=0.949)$. Based on the PointBreak trial, we have CarPacBev (Carboplatin-Paclitaxel-Bevacizumab) as the most effective evidence-based platinum-based doublet combination with bevacizumab (4).

The question of whether the addition of immunotherapy to bevacizumab plus chemotherapy doublet improved outcomes was addressed by the IMpower 150 trial. The IMpower150 showed that the addition of atezolizumab (anti-PD-L1) to bevacizumab + carboplatin + paclitaxel (Atezo-CarPacBev) induction followed by maintenance atezolizumab + bevacizumab improved outcomes compared

^ ORCID: Meghana Kesireddy, 0000-0002-9248-2603; Apar Kishor Ganti, 0000-0003-3724-2671. 
to bevacizumab + carboplatin + paclitaxel (CarPacBev) induction followed by maintenance bevacizumab (or) atezolizumab + carboplatin + paclitaxel (Atezo-CarPac) induction followed by maintenance atezolizumab in metastatic or recurrent non-squamous NSCLC. The PFS and OS benefit of the quadruplet regimen (AtezoCarPacBev) was seen irrespective of PD-L1 status and even in patients with EGFR/ALK alterations. This trial showed improved outcomes with the addition of atezolizumab (immunotherapy) to bevacizumab plus chemotherapy doublet, which could be due to the reversal of VEGF mediated immunosuppression by bevacizumab leading to enhanced efficacy of atezolizumab. In terms of safety, quadruplet therapy had indeed higher toxicity compared to triplet therapy $(5,6)$.

The other trial which assessed the quadruplet therapy in advanced non-squamous NSCLC is a single-arm, phase 2 clinical trial (Big Ten Cancer Research Consortium Study) that assessed if the addition of atezolizumab to the triplet combination of carboplatin, pemetrexed and bevacizumab would lead to better PFS. This study was closed early due to 3 possibly treatment-related deaths (likely due to bevacizumab). However, the 1-year PFS and 1 year OS were $55.27 \%$ and $82.9 \%$, respectively, which is longer than the historical control (of Atezo-CarPacBev); however, these positive results need to be further explored in a phase 3 trial (7).

Of note, there are no randomized controlled trials that directly compared carboplatin-based chemotherapy against cisplatin-based chemotherapy in NSCLC. The meta-analysis by Griesinger et al. (included a total of 12 randomized controlled trials) compared the efficacy and safety of carboplatin versus cisplatin-based chemotherapy in patients with advanced NSCLC showed no significant difference in OS between carboplatin vs. cisplatin-based chemotherapy (HR 1.08 with 95\% CI: 0.96-1.21), but a slight benefit in ORR with cisplatin (RR $0.88,95 \%$ CI: 0.78-0.99) (8).

The CLEAR (phase 2) trial by Udagawa et al. was designed to find the most effective platinum-based combination with bevacizumab. The final goal of the authors was to determine the most effective platinum-based bevacizumab to be combined with atezolizumab. Patients with advanced (stage IIIB or IV) or recurrent non-squamous NSCLC were randomly assigned in a 2:1 ratio to either cisplatin + pemetrexed + bevacizumab (CisPemBev) followed by maintenance pemetrexed + bevacizumab (PemBev) or Carboplatin + paclitaxel + bevacizumab (CarPacBev) followed by maintenance bevacizumab (Bev). This trial showed that the primary endpoint of PFS was better in the CisPemBev (7.6 months) compared to CarPacBev (7 months) [HR of 0.825 (95\% CI: 0.600-1.134)]. The OS was, however, not statistically different in the CisPemBev (23.4 months) compared to CarPacBev (21.6 months), HR 0.845 (95\% CI: $0.58-1.24)$. The overall response rate was similar with $57 \%$ in the CisPemBev group and $55 \%$ in the CarPacBev group. In terms of safety, CisPemBev had a better safety profile with $67 \% \geq$ grade 3 adverse events in CisPemBev group vs. $82 \%$ in $\mathrm{CarPacBev}$ group.

The CLEAR trial compared the cisplatin-based regimen (CisPemBev) against the carboplatin-based regimen (CarPacBev). The POINTBREAK trial (4) already showed that pemetrexed was not superior to paclitaxel in terms of OS but had better PFS when combined with carboplatinbevacizumab. Hence it is unclear if the PFS benefit seen with CisPemBev in the CLEAR trial is due to the cisplatin or pemetrexed. Also, part of the PFS improvement seen in the CLEAR trial could be because the maintenance arm of CisPemBev had pemetrexed + bevacizumab as opposed to only bevacizumab in the CarPacBev arm. Both ECOGACRIN 5508 and AVAPERL trials showed that combination (pemetrexed + bevacizumab) maintenance led to better PFS than single-agent bevacizumab maintenance alone in advanced non-squamous NSCLC. In ECOG-ACRIN 5508, maintenance therapy was given after 4 cycles of induction with carboplatin + paclitaxel + bevacizumab (same as one arm of the CLEAR trial). The PFS was better in bevacizumab + pemetrexed maintenance compared to bevacizumab maintenance but OS was similar in both arms (9). Similarly, in AVAPERL, phase 3 trial, maintenance therapy was given after 4 cycles of induction with cisplatin + pemetrexed + bevacizumab (same as the other arm of the CLEAR trial). The bevacizumab + pemetrexed maintenance improved PFS but not OS compared to pemetrexed maintenance alone (10). In both trials, the bevacizumab + pemetrexed combination was associated with higher toxicity.

In the CLEAR study, the grade 1-2 adverse events data was collected only for bevacizumab and not for chemotherapeutic agents. Though the overall incidence of $\geq$ grade 3 adverse events was lower in the CisPemBev group in the CLEAR trial, it is to be noted that the side effect profile is different in both these groups with a higher incidence of hematological toxicity with carboplatin and higher incidence of non-hematological toxicity with cisplatin similar to the findings in the meta-analysis by Griesinger et al. (8). In the meta-analysis, the risk of anemia (RR 3.94) and the risk of thrombocytopenia (RR 2.46) was 
higher with carboplatin, while the risk of nausea/vomiting (RR 0.53) was higher with cisplatin. Given the different toxicity profiles, patients' co-morbidities, symptoms, and preferences (impact of the toxicity on patients' quality of life) usually play a crucial role in selecting cisplatin versus carboplatin-based regimens.

The CLEAR trial showed improvement in PFS, but not OS. This would suggest that the possibility of a major difference between the two regimens is relatively low. The results of the Big Ten study suggest the possibility of increased toxicity with the four-drug combination, and that should be taken into account when designing future trials assessing the efficacy of CisPemBev combination with atezolizumab. The bar to establish the CisPemBevatezolizumab combination as the new standard of care is quite high. This would need a large, randomized phase 3 trial and unlikely to change the current standard of either carboplatin-pemetrexed-pembrolizumab or carboplatinpaclitaxel-bevacizumab-atezolizumab.

\section{Acknowledgments}

Funding: None.

\section{Footnote}

Provenance and Peer review: This article was commissioned by the editorial office, Translational Lung Cancer Research. The article did not undergo external peer review.

Conflicts of Interest: Both authors have completed the ICMJE uniform disclosure form (available at https://dx.doi. org/10.21037/tlcr-21-718). Dr. AKG reports consulting fees from AstraZeneca, Cardinal Health, Blueprint Medicines, G1 Therapeutics, Mirati Therapeutics and Flagship Biosciences; institutional grants from Apexigen, Merck, Nektar Therapeutics, TAB Biosciences, Novartis, WindMil Therapeutics and Takeda; participated in Data Safety Monitoring Boards for YMabS Therapeutics and Hoosier Cancer Research Network. The other author has no conflicts of interest to declare.

Ethical Statement: The authors are accountable for all aspects of the work in ensuring that questions related to the accuracy or integrity of any part of the work are appropriately investigated and resolved.

Open Access Statement: This is an Open Access article distributed in accordance with the Creative Commons Attribution-NonCommercial-NoDerivs 4.0 International License (CC BY-NC-ND 4.0), which permits the noncommercial replication and distribution of the article with the strict proviso that no changes or edits are made and the original work is properly cited (including links to both the formal publication through the relevant DOI and the license). See: https://creativecommons.org/licenses/by-nc-nd/4.0/.

\section{References}

1. Gandhi L, Rodríguez-Abreu D, Gadgeel S, et al. Pembrolizumab plus Chemotherapy in Metastatic NonSmall-Cell Lung Cancer. N Engl J Med 2018;378:2078-92.

2. Sandler A, Gray R, Perry MC, et al. Paclitaxel-carboplatin alone or with bevacizumab for non-small-cell lung cancer. N Engl J Med 2006;355:2542-50.

3. Al-Saleh K, Quinton C, Ellis PM. Role of pemetrexed in advanced non-small-cell lung cancer: meta-analysis of randomized controlled trials, with histology subgroup analysis. Curr Oncol 2012;19:e9-e15.

4. Patel JD, Socinski MA, Garon EB, et al. PointBreak: a randomized phase III study of pemetrexed plus carboplatin and bevacizumab followed by maintenance pemetrexed and bevacizumab versus paclitaxel plus carboplatin and bevacizumab followed by maintenance bevacizumab in patients with stage IIIB or IV nonsquamous non-small-cell lung cancer. J Clin Oncol 2013;31:4349-57.

5. Socinski MA, Jotte RM, Cappuzzo F, et al. Atezolizumab for First-Line Treatment of Metastatic Nonsquamous NSCLC. N Engl J Med 2018;378:2288-301.

6. Socinski MA, Nishio M, Jotte RM, et al. IMpower150 Final Overall Survival Analyses for Atezolizumab Plus Bevacizumab and Chemotherapy in First-Line Metastatic Nonsquamous NSCLC. J Thorac Oncol 2021. [Epub ahead of print]. doi: 10.1016/j.jtho.2021.07.009. J Clin Oncol 2021;39:9034.

7. Ardeshir-Larijani F, Althouse SK, Leal T, et al. Phase II trial of atezolizumab (A) + carboplatin (C) + pemetrexed (P) + bevacizumab (B) in pts with stage IV non-squamous nonsmall cell lung cancer (NSq-NSCLC): Big Ten Cancer Research Consortium Study LUN 17-139.

8. Griesinger F, Korol EE, Kayaniyil S, et al. Efficacy and safety of first-line carboplatin-versus cisplatin-based chemotherapy for non-small cell lung cancer: A metaanalysis. Lung Cancer 2019;135:196-204.

9. Ramalingam SS, Dahlberg SE, Belani CP, et al. Pemetrexed, Bevacizumab, or the Combination As 
Maintenance Therapy for Advanced Nonsquamous NonSmall-Cell Lung Cancer: ECOG-ACRIN 5508. J Clin Oncol 2019;37:2360-7.

10. Barlesi F, Scherpereel A, Gorbunova V, et al. Maintenance bevacizumab-pemetrexed after first-line cisplatin-

Cite this article as: Kesireddy M, Ganti AK. Cisplatin or carboplatin for advanced non-small cell lung cancer: does it really matter? Transl Lung Cancer Res 2021;10(9):3705-3708. doi: $10.21037 /$ tlcr-21-718 pemetrexed-bevacizumab for advanced nonsquamous nonsmall-cell lung cancer: updated survival analysis of the AVAPERL (MO22089) randomized phase III trial. Ann Oncol 2014;25:1044-52. 